\title{
Highlights from the Telescope Array experiment
}

\section{Grigory I. Rubtsov ${ }^{a * *}$ on behalf of the Telescope Array Collaboration ${ }^{\dagger}$}

${ }^{a}$ Institute for Nuclear Research of the Russian Academy of Sciences, 60th October Anniversary st. 7A, Moscow, Russia

E-mail: grisha@ms2.inr.ac.ru

The Telescope Array (TA) is the largest cosmic ray observatory in the Northern Hemisphere. It is designed to measure the properties of cosmic rays over a wide range of energies. TA with it's low energy extension (TALE) observe cosmic-ray induced extensive air showers between $2 \times 10^{15} \mathrm{eV}$ and $2 \times 10^{20} \mathrm{eV}$ in hybrid mode using multiple instruments, including an array of scintillator detectors at the Earth's surface and telescopes to measure the fluorescence and Cerenkov light. The statistics at the highest energies is being enhanced with the ongoing construction of the TAx4 experiment which will quadruple the surface area of the detector. We review the present status of the experiments and most recent physics results on the cosmic ray anisotropy, chemical composition and energy spectrum. Notable highlights include a new feature in the energy spectrum at about $10^{19.2} \mathrm{eV}$, and a new clustering of events in the direction of Perseus-Pisces supercluster above this energy. We also report on updated diffuse photon flux limits and new spectrum and composition results in the lower energy range from the TALE extension.

$37^{\text {th }}$ International Cosmic Ray Conference (ICRC 2021)

July 12 th - 23rd, 2021

Online - Berlin, Germany

\footnotetext{
${ }^{*}$ Presenter

${ }^{\dagger}$ A complete list of authors can be found at the end of the paper
} 


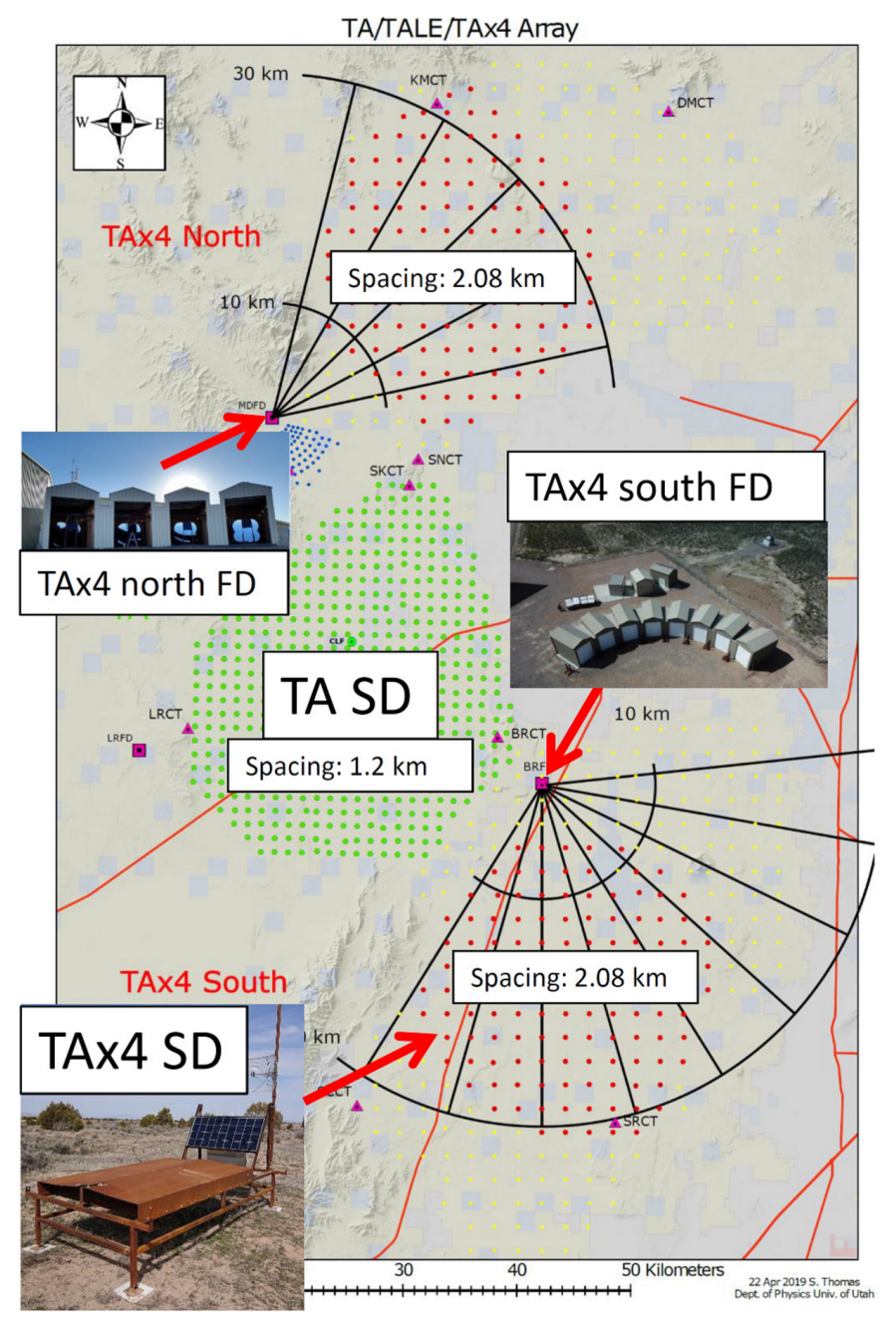

Figure 1: General view of the Telescope Array (TA) Observatory. Red and yellow circles show deployed and not yet deployed TAx4 SDs. Positions of TALE SDs are marked with blue circles. The fields of view of TAx4 FDs are shown with black shapes [5].

\section{Introduction}

The Telescope Array (TA) experiment is a hybrid detector operating in Utah, USA since 2008. The TA Surface Detector (TA SD) [1] is an array of 507 solar-powered stations covering an area of $680 \mathrm{~km}^{2}$ placed in a square grid with $1.2 \mathrm{~km}$ spacing. Each SD station contains two layers of a plastic scintillator with an area of $3 \mathrm{~m}^{2}$. The surface detector is overlooked by three fluorescence detector (FD) stations - Middle Drum, Black Rock Mesa and Long Ridge with a total of 36 fluorescence telescopes [2].

The TA is the largest cosmic ray observatory in the Northern Hemisphere. Its primary science goal is the origin and properties of the ultrahigh-energy cosmic rays. These implies the study of the spectrum, composition and anisotropy of the arrival directions of cosmic rays in a wide range of energies. More scientific tasks include the physics of high-energy hadronic interactions, 
multi-messenger and interdisciplinary studies.

There are two extensions of the TA experiment, namely TA Low-energy Extension (TALE) and high energy extension TAx4. Both extensions are hybrid experiments like TA. The TALE FD station located at TA Middle Drum site has ten fluorescence telescopes, which observe $31-59^{\circ}$ in elevation, on top of the field of view of Middle Drum FD station. The TALE SD array consists of 80 stations, half of which are located with $400 \mathrm{~m}$ spacing and the other half with $600 \mathrm{~m}$ spacing. It is planned to further enhance TALE SD by installing additional 54 stations with $100 \mathrm{~m}$ and $200 \mathrm{~m}$ spacing [3]. TALE SD and TALE FD are in operation since September 2013 and September 2018, correspondingly. Operation of the TALE extension made it possible to extend the available energy range down to $2 \mathrm{PeV}[4]$.

The high energy extension TAx4 is designed to increase the rate of the data collection at the highest energies. The spacing of the TAx $4 \mathrm{SD}$ array is $2.08 \mathrm{~km}$ which makes it efficient for cosmic rays with energies greater than $57 \mathrm{EeV}$. The area covered by $500 \mathrm{TAx} 4 \mathrm{SD}$ stations is approximately three times larger area than the one covered by TA SD. The combined effective area of the TA SD and TAx4 SD is approximately $2800 \mathrm{~km}^{2}$. Two TAx4 FD stations are designed to observe the hybrid events which are detected by both SDs and FDs. The construction of the TAx4 FD is finished and more than half of the TAx4 SDs (257 SDs) have been deployed in 2019, see Figure 1. The observations of TAx4 in hybrid mode have started in June 2020 [5, 6].

The paper is organized as follows. The selected results on the TA and TALE energy spectrum are presented in Section 2, including declination dependence of the TA spectrum. The Section 3 shows the results on TA and TALE hybrid composition as well as the TA SD composition. The new ultrahigh-energy photon flux limits are given in Section 4. The results on the arrival direction anisotropy are highlighted in Section 5. These include the dipole update, hot spot update, new clustering of high-energy events in the direction of Perseus-Pisces supercluster, and chemical composition constraints from the anisotropy. Two interdisciplinary results, namely observations of the Terrestrial Gamma-Ray Flashes with TA SD and variations of the SD trigger rate during Thunderstorms are explained in Section 6. The results are summarized in Section 7.

\section{Energy spectrum}

The TA observatory has measured the cosmic ray spectrum over unprecedented five decades of energy from $2 \mathrm{PeV}$ to $200 \mathrm{EeV}$. Figure 2 shows the TA combined energy spectrum produced using the 22 months TALE measurements in monocular mode [4] below $10^{18.2} \mathrm{eV}$ and an 11 year TA SD spectrum [7] above $10^{18.2} \mathrm{eV}$. The combined TA spectrum exhibits the knee feature at about $10^{15.5} \mathrm{eV}$, the low energy ankle feature at $10^{16.22 \pm 0.02} \mathrm{eV}$, the second knee at $10^{17.04 \pm 0.04} \mathrm{eV}$, the ankle at $10^{18.69 \pm 0.01} \mathrm{eV}$, and the cutoff at $10^{19.81 \pm 0.03} \mathrm{eV}$ [8]. Preliminary energy spectrum above $10 \mathrm{EeV}$ measured by the TAx4 SD is compatible with the TA SD spectrum [9].

The Pierre Auger Collaboration has reported a new feature in the cosmic ray spectrum at energy close to $10^{19.1} \mathrm{eV}$, named "instep" [10]. We have conducted a search for this new feature by combining the HiRes I monocular [11], TA FD Black Rock and Long Ridge monocular [12] and TA SD [7] spectrum measurements. The joint fit of these three spectra finds the "instep" feature at $10^{19.25 \pm 0.03}$ with a statistical significance of 5.3 standard deviations [8]. 


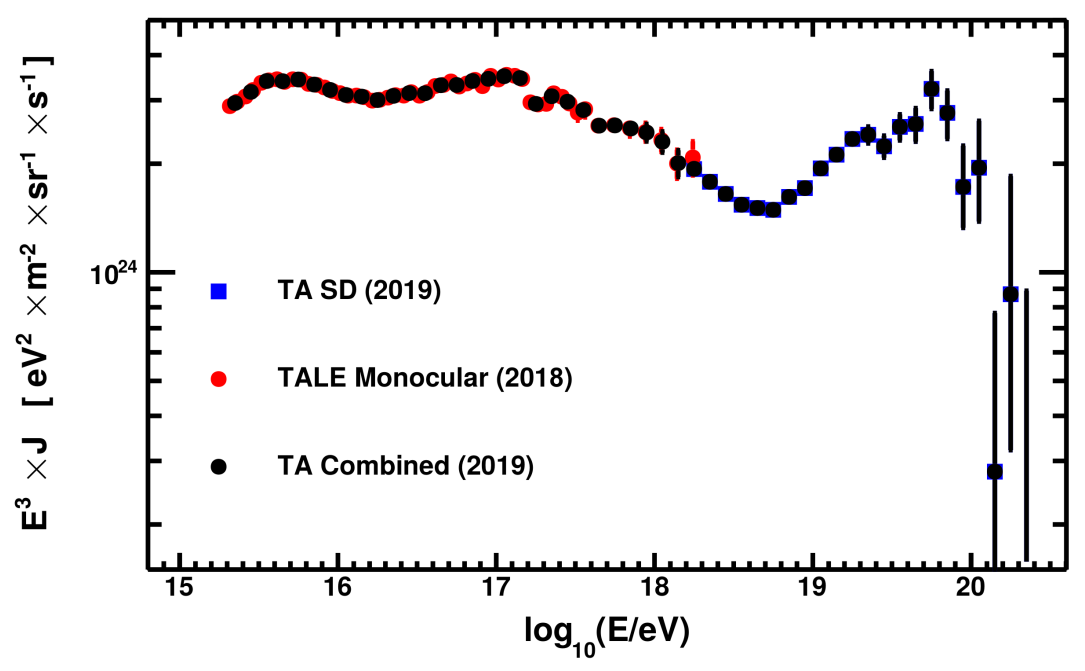

Figure 2: The TA combined energy spectrum (black points) composed of 11 years of the TA SD data (blue squares) and 22 months of TALE monocular data (red circles) [8].

\begin{tabular}{ccc} 
Parameter & Auger & TA \\
\hline$\gamma_{1}$ & $3.29 \pm 0.02$ & $3.23 \pm 0.01$ \\
$\gamma_{2}$ & $2.51 \pm 0.03$ & $2.63 \pm 0.02$ \\
$\gamma_{3}$ & $3.05 \pm 0.05$ & $2.92 \pm 0.06$ \\
$\gamma_{4}$ & $5.1 \pm 0.3$ & $5.0 \pm 0.4$ \\
$E_{\text {ankle }} / \mathrm{EeV}$ & $5.0 \pm 0.1$ & $5.4 \pm 0.1$ \\
$E_{\text {instep }} / \mathrm{EeV}$ & $13 \pm 1$ & $18 \pm 1$ \\
$E_{\text {cut }} / \mathrm{EeV}$ & $46 \pm 3$ & $71 \pm 3$
\end{tabular}

Table 1: The fit parameters of the Auger and TA spectra: four power-law indices and three break points $E_{\text {ankle }}, E_{\text {instep }}, E_{\text {cut }}$. The errors quoted are the statistical errors [13].

The results of the joint Auger and TA spectrum working group presented at the Conference [13] demonstrate that the spectra of two experiments are compatible in the energy range $E<10^{19} \mathrm{eV}$ with a $9 \%$ difference in the absolute energy scale. Still, a discrepancy persists at the highest energies. Two spectra agree in the common declination band $-15.7^{\circ}<\delta<24.8^{\circ}$ with an additional assumption of the energy dependent shift $\pm 10 \%$ per decade for energies greater that $10^{19} \mathrm{eV}$. The comparison of the broken power-law fits for Auger and TA spectra is given in Table 1 [13].

It is shown that the TA SD spectrum is considerably different in the two declination bands, above and below $\delta=24.87^{\circ}$. The high-energy cut-off appears at $10^{19.64 \pm 0.04}$ and $10^{19.84 \pm 0.02} \mathrm{eV}$ in the lower and higher band, correspondingly. The global significance of the difference of the spectra is 4.3 standard deviations [14]. 

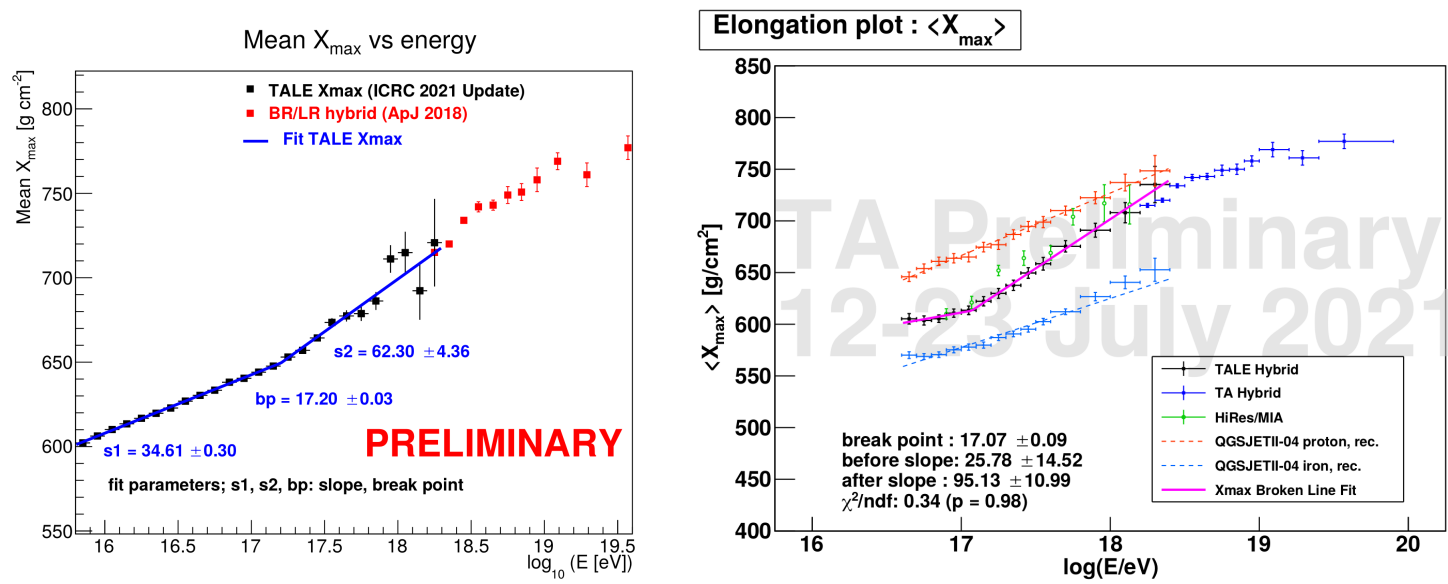

Figure 3: Mean reconstructed depth of the shower maximum $X_{\max }$ as a function of shower energy measured by (left) TALE FD monocular (black points) [15] and TA FD monocular (red points) [18]; (right) TALE Hybrid detector [16].
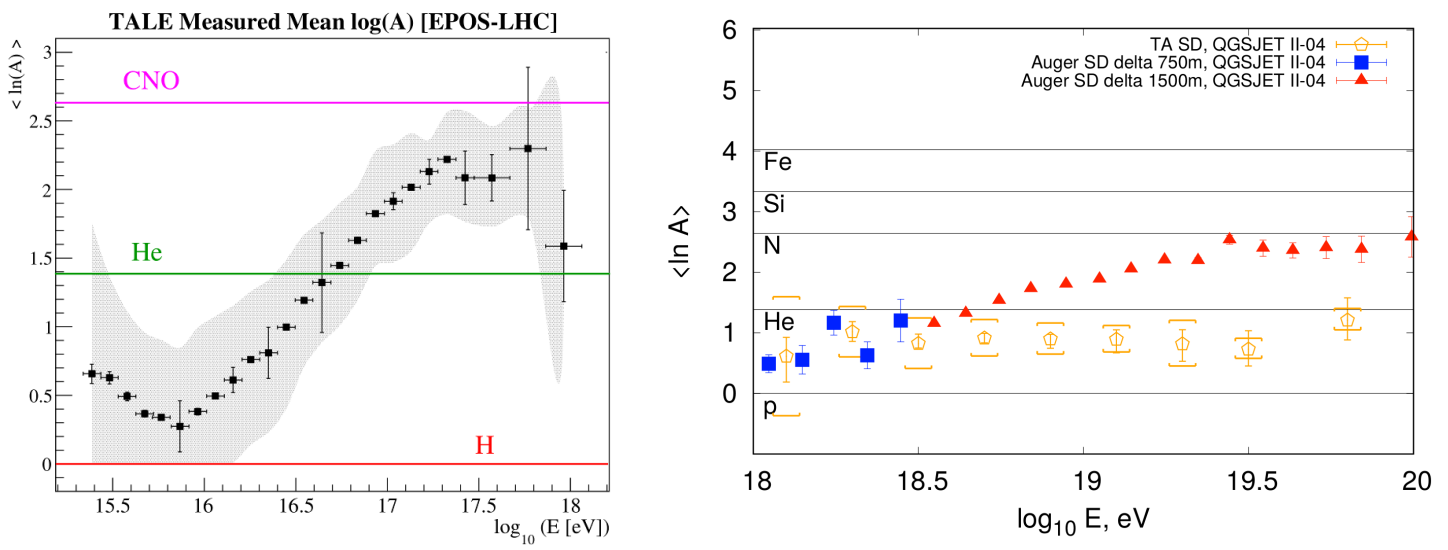

Figure 4: Mean logarithmic atomic mass as a function of energy measured by (left) TALE FD monocular; (right) TA SD (orange pentagons) [20] compared with the Pierre Auger Observatory SD delta results (blue squares and red triangles) [24].

\section{Chemical composition and hadronic interactions}

The cosmic-ray composition is measured at TA Observatory in the energy range from $3 \mathrm{PeV}$ to $60 \mathrm{EeV}$. These include the $X_{\max }$ measurements of TALE FD [15-17] in the lower energy range from $3 \mathrm{PeV}$ to $1 \mathrm{EeV}$, the TA FD $X_{\max }$ results $[18,19]$ for energies greater than $0.3 \mathrm{EeV}$ and the TA SD composition at the highest energies up to $60 \mathrm{EeV}$ obtained with the multivariate analysis using Boosted Decision Trees [20, 21].

The Figure 3 shows so-called elongation plot - mean reconstructed depth of the shower maximum $X_{\max }$ as a function of shower energy measured by TALE FD in both monocular mode [15] and hybrid mode [16] and TA FD [18]. The elongation rate demonstrates a break at energy of 


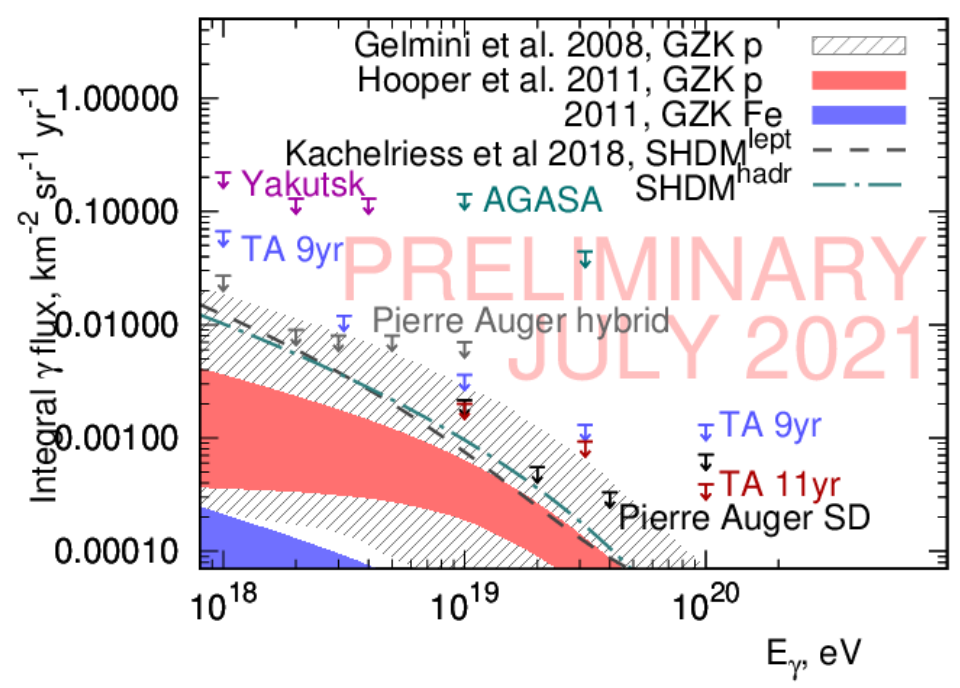

Figure 5: The TA SD photon flux limits presented at the Conference [29] based on the neural network p- $\gamma$ classifier compared with the results from AGASA [32], Pierre Auger Observatory [33-35], Yakutsk [36], and previous TA SD result [31]. The predictions of certain models are shown with lines and shaded areas [37-39].

$\sim 10^{17.2} \mathrm{eV}$ which is correlated with the observed break - second knee - in the cosmic rays energy spectrum. The mean logarithmic atomic mass measured by TALE FD in monocular mode and TA SD is shown in Figure 4. The TALE results shows that the composition gets heavier between first and second knees, which is consistent with Peters Cycle interpretation of the cosmic ray spectrum [22]. The latter implies that galactic sources have the maximum acceleration energy of cosmic rays, approximately proportional to the nuclear charge, see e.g. [23] for a review. As one may see in Figure 4 (right) at the highest energies the results of TA SD indicate light composition, which is not consistent with the Auger SD results showing heavier composition [24].

The joint fit of the TA spectrum and composition result within the astrophysical model of ultrahigh-energy cosmic-ray sources is presented [25]. The model results in the prediction of the cosmogenic neutrino flux, which is considerably higher than one predicted by similar fits to Auger data.

The $X_{\max }$ for the ultrahigh-energy events carries important information on the proton-air cross section. Namely, it is shown that the cross-section is directly related with the exponential decay scale of the tail of the $X_{\max }$ distribution [26]. Moreover, the information on the chemical composition, e.g. proton-to-helium ration, may be derived from the same decay scale value [27]. The results of the proton-air cross-section measurements at $\sqrt{s}=73 \mathrm{TeV}$ using the data of TA Black Rock (BR) and Long Ridge (LR) FD collected in hybrid mode are presented at the Conference [28]. The proton-air cross-section is observed to be $520.1 \pm 35.8$ [Stat. $]_{-42.9}^{+25.3}$ [Sys.] mb. The result is in a reasonable agreement both with the results of HiRes and Auger experiments and the prediction of high-energy hadronic models [28]. 

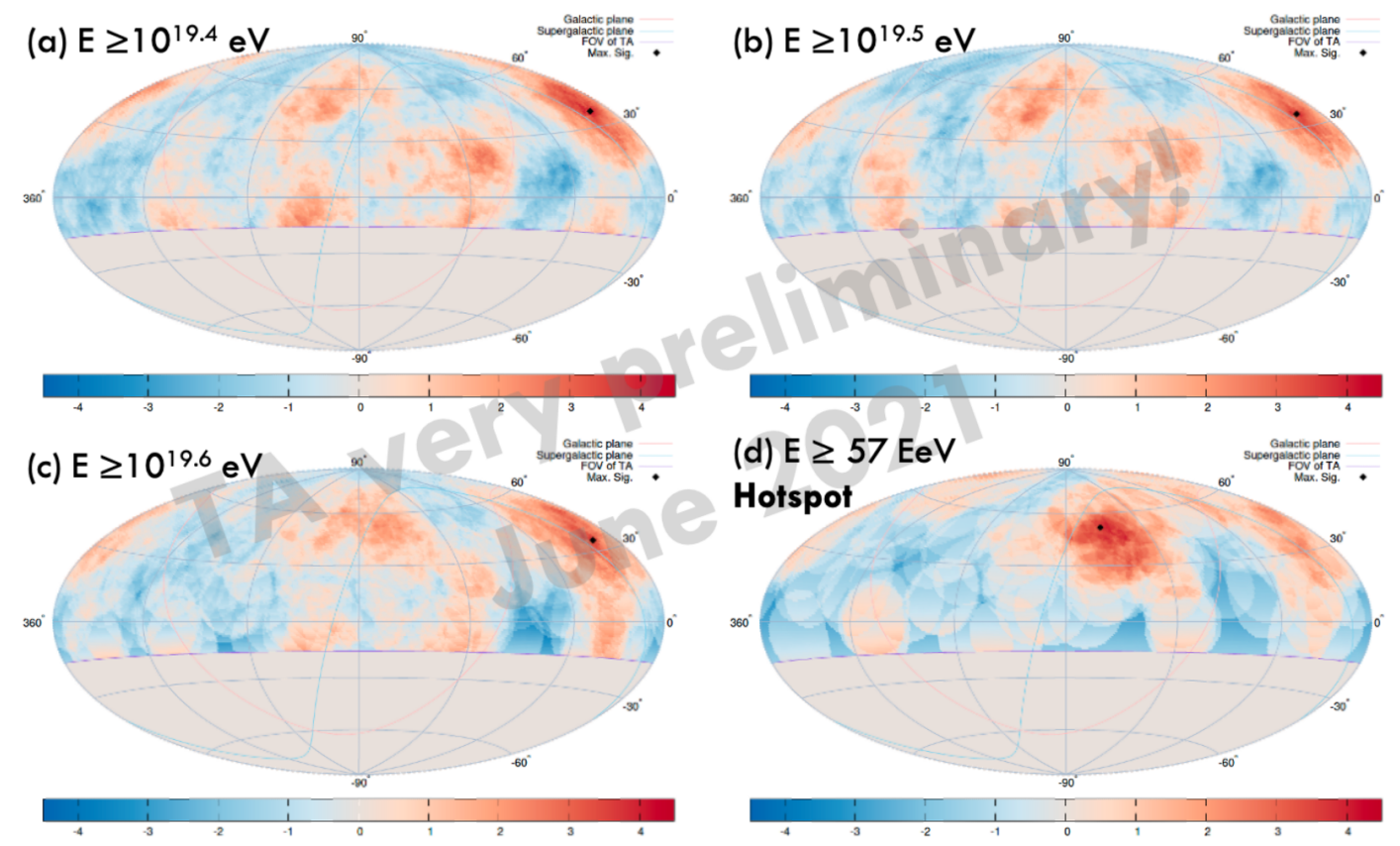

Figure 6: The maps of the Li-Ma significance of excess and deficit for 4 energy thresholds $E>10^{19.4} \mathrm{eV}$ (a), $E>10^{19.5} \mathrm{eV}$ (b), $E>10^{19.6} \mathrm{eV}$ (c), and $E>57 \mathrm{EeV}$ (d). The black dot shows the position of maximum Li-Ma significance.

\section{Photon search}

The updated results of the search for the ultrahigh-energy photons with primary energies greater than $10 \mathrm{EeV}$ are presented [29]. The analysis is based on the neural network classifier which employs full time-resolved signals from all triggered TA SD stations along with 16 composition-sensitive observables [30]. Implementation of the new classifier have substantially increased the efficiency of the photon candidate selection compared to the previous analysis with the classifier based on the Boosted Decision Trees [31]. The TA SD diffuse photon flux limits compared with the results of Pierre Auger, Yakutsk and AGASA experiments are shown in Figure 5.

\section{Anisotropy results}

This section features the main TA results on the cosmic-ray anisotropy. Plese refer to the "TA Anisotropy Summary" talk at the Conference for a more detailed review of these results [40].

The Pierre Auger Collaboration in 2017 has reported observation of the dipole-type anisotropy in the arrival direction of the cosmic rays with energies above $8 \mathrm{EeV}$ [41]. We have performed the search of dipole structure in the latest data set of the TA SD. We have restricted the analysis on the same energy threshold used by Auger. The latter corresponds to the condition $E_{\mathrm{TA}}>8.8 \mathrm{EeV}$ with the account of the $10 \%$ difference in the energy scale between TA and Auger experiments [42]. The amplitude and phase of the dipole reconstructed by TA SD are compatible with the corresponding 
values reported by Auger. Still, with the present statistics the TA SD result is consistent with the fluctuation of isotropic model at $2 \sigma$ level [43].

The full sky analysis of dipole and quadrupole have been performed in three energy ranges (8-16 EeV, 16-32 EeV and 32- $\infty \mathrm{EeV}$ ) using the combined Auger SD and TA SD dataset [44]. The use of the full sky data results in substantially smaller error in determining the components of both dipole and quadrupole.

In 2014 the TA experiment observed a cluster of high energy events $(E>57 \mathrm{EeV})$ in a circle of $20^{\circ}$ radius centered at R.A. $=146.7^{\circ}, \delta=43.2^{\circ}$. The number of events in the circle was found to be 19 out of 72 total with the expectation of 4.5 events in case of uniform background. The post-trial significance of the "hot spot" was reported at the level of $3.4 \sigma$ [45]. We have repeated the search for the hot spot above $57 \mathrm{EeV}$ using 12 years of TA SD data. This represents a significant increase of the statistics compared to the 5 years in the original analysis. The most significant excess have been observed in a circle of $25^{\circ}$ radius centered at R.A. $=140.0^{\circ}, \delta=40.5^{\circ} .40$ of 179 events are located inside the circle, whereas only 14.6 events are expected in case of isotropic distribution. The post-trial significance has decreased to $3.2 \sigma$, while the increase rate of the events inside the new hot spot circle is consisted with linear law within $1 \sigma$ [46].

The search for the event concentration at lower energies $E>10^{19.4} \mathrm{eV}, E>10^{19.5} \mathrm{eV}$, and $E>10^{19.6} \mathrm{eV}$ has revealed new excess in the direction of Perseus-Pisces supercluster. The strongest excess has been observed for $E>10^{19.4} \mathrm{eV}$ at R.A. $=17.4^{\circ}, \delta=36.0^{\circ}$ with a local Li-Ma significance of about 4 standard deviations, see Figure 6 [46].

The distribution of the arrival directions of cosmic rays can serve as a measure of the cosmic ray mass composition. The new method has recently been developed for this purpose [47]. The method constructs a test statistic (TS) based on the characteristic deflection of ultrahigh-energy cosmic rays with respect to the luminous matter distribution in the local Universe. It is shown that the TS is sensitive to the mass composition of the cosmic-ray events and the results of the analysis are stable with respect to the model of galactic magnetic field. The method is applied to the TA SD data and the new independent constraints on the proton and iron fractions are derived. The results favor growth of the proton fraction and corresponding decrease of the iron fraction at $10<E<100 \mathrm{EeV}$ and pure iron at the energies above $100 \mathrm{EeV}$ [48]. It is also found that the Auger best-fit composition model [49] is in tension with TA SD arrival directions at the level of $2 \sigma$ [48].

\section{Interdisciplinary results}

\subsection{Observation of Terrestrial Gamma-Ray Flashes with the TA SD}

The studies of energetic radiation from lightnings at TA started with the observation of the short-time bursts of TA SD events associated with the lightning flashes [50]. The installation of a very high frequency Lightning Mapping Array (LMA) and electric field sensors in 2014 confirmed that these events were indeed downward terrestrial gamma-ray flashes (TGFs) initiated in the first couple milliseconds of negative lightning flashes [51]. A broadband interferometer (INTF) has been recently added to TA site, turning the latter into versatile instrument for TGF studies.

We report observation of four TFGs, three of which result from negative cloud-to-ground strokes and the fourth results from negative intracloud flash. It is clearly seen that the onset of each 


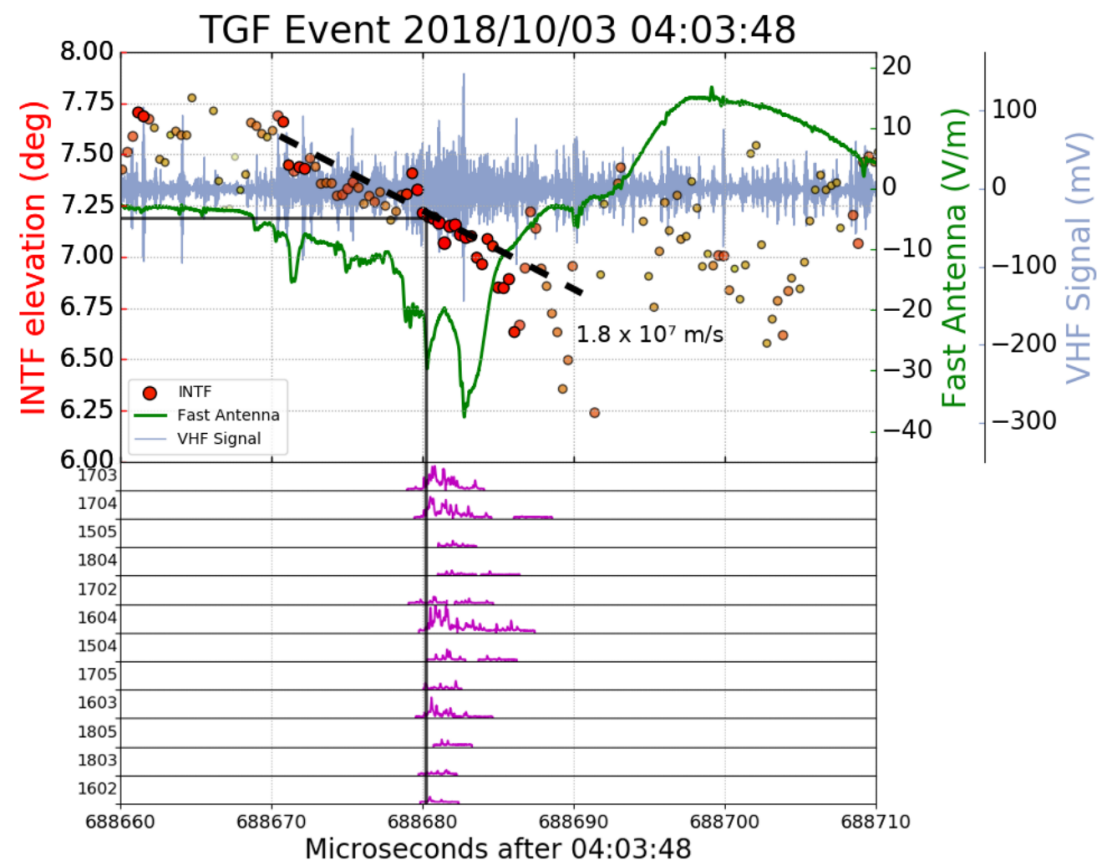

Figure 7: One of the TGFs registered at Telescope Array Observatory. The responses of TA SD stations are depicted by purple curves. INTF data are shown with circles with color, size and opacity representing power. Raw very high frequency LMA waveform is represented with light blue line. Green curve shows the electric field change recorded by the fast sferic sensor [52].

of these TGFs coincide with the strong initial breakdown pulses (IBPs). The latter produces strong, impulsive signals in the electric field change data, recorded by the fast sferic sensor. Breakdown activity is also mapped by the LMA and by the INTF, see Figure 7 [52].

\subsection{Observation of variations of SD trigger rate during Thunderstorms and Implications for Large-Scale Electric Field}

There are three trigger levels for TA SD, see [1] for details. The level-0 trigger is activated when the scintillation energy greater than 0.3 Minimum Ionizing Particles (MIP) is recorded at particular SD station. The level-0 trigger rate (approximately $750 \mathrm{~Hz}$ ) is monitored at each station with 10 minutes resolution and is included in the calibration data set.

We have investigated the thunderstorms reported by the National Lightning Detection Network which have high recorded peak currents $(>90 \mathrm{kA}$ ). It is shown that the deficit and excess of trigger rate is observed at the level of $\pm 1-2 \%$ in the time periods in which the thunderstorm passes above the TA SD. The simulations are performed with the EFIELD option of CORSIKA [53] for large-scale electric field using the SYBILL and URQMD for the high and low energy hadronic interaction models, correspondingly.

In order to reproduce the observations we have performed simulations of the cosmic ray showers assuming two models of electric field. The field is assumed constant and vertically directed. In the first model the field is localized inside the cloud, while in the second model it covered the 
entire distance from cloud to groud. The total potential difference varies in simulations between $-0.4 \mathrm{GV}$ and $0.4 \mathrm{GV}$. It is shown that the first model can predict both deficit and excess of trigger rate depending on the potential difference, while the second model does always predict excess. The interpretation of the observations is therefore dependent on both the polarity and the type of the thunderstorm (intra-cloud or cloud-to-ground). In order to proceed with more detailed studies of the thunderstorm structure we plan to install the array of electric field mills at the Telescope Array site [54].

\section{Summary}

As a summary we briefly list the most important results of the Telescope Array ultra-high energy cosmic ray Observatory presented at the Conference:

- The construction of the TAx4 FD is finished and more than half of the TAx4 SDs (257 SDs) have been deployed in 2019 .

- The cosmic ray energy spectrum is measured over an unprecedented wide energy range from $10^{15.5}$ to $10^{20.5} \mathrm{eV}$.

- The new "instep" feature in the energy spectrum is confirmed at energy close to $10^{19.25} \mathrm{eV}$.

- According to the results of TALE FD, the cosmic ray composition is becoming heavier between the first and the second knees.

- Both the composition and positions of the first and the second knees in the spectrum $\left(10^{15.6} \mathrm{eV}\right.$ and $10^{17.1} \mathrm{eV}$ ) are consistent with the Peters Cycle interpretation.

- Both the TA FD hybrid $X_{\max }$ measurements and TA SD composition results are compatible with predominantly light elements such as protons and helium between $10^{18.0} \mathrm{eV}$ and $10^{19.1} \mathrm{eV}$.

- The proton-air cross-section is estimated as $520.1 \pm 35.8[\text { Stat. }]_{-42.9}^{+25.3}[$ Sys.] mb at $\sqrt{s}=73 \mathrm{TeV}$ using the TA FD data in hybrid mode.

- The diffuse photon flux limits are significantly strengthened using novel neural network classifier.

- There are several indications of the anisotropy of arrival directions at the highest energy:

- Hot spot in the direction of Ursa Major (3.2 $\sigma$ post trial).

- Hint of the excess in the direction of Perseus-Pisces supercluster for $E>10^{19.3} \mathrm{eV}$.

- Declination dependence of the spectrum.

- The method for estimating cosmic-ray composition based on the correlations of the arrival directions with the luminous matter distribution in the local Universe has been developed. As a result of applying the method to TA SD data a composition with a large fraction of protons is obtained. 
- Multiple TGFs have been observed with the TA SD together with Lightning Mapping Array and broadband interferometer. It is shown that the onset of the TGF coincides with the strong initial breakdown pulses.

- It is shown that the thunderstorm activity affects the trigger rate of TA SD. The amplitude of the trigger rate change depends on both the polarity and the type of the thunderstorm.

\section{References}

[1] T. Abu-Zayyad et al. [Telescope Array Collaboration], Nucl. Instrum. Meth. A 689, 87 (2012).

[2] H. Tokuno et al. [Telescope Array Collaboration] Nucl. Instrum. Meth. A 676, 54 (2012).

[3] S. Ogio et al. [Telescope Array Collaboration] PoS ICRC 2021, 255 (2021).

[4] R. U. Abbasi et al. [Telescope Array], Astrophys. J. 865, no.1, 74 (2018).

[5] E. Kido et al. [Telescope Array Collaboration] PoS ICRC 2021, 203 (2021).

[6] S. Kim et al. [Telescope Array Collaboration] PoS ICRC 2021, 332 (2021).

[7] D. Ivanov et al. [Telescope Array Collaboration] PoS ICRC 2019, 298 (2019).

[8] D. Ivanov et al. [Telescope Array Collaboration] PoS ICRC 2021, 341 (2021).

[9] H. Jeong et al. [Telescope Array Collaboration] PoS ICRC 2021, 331 (2021).

[10] A. Aab et al. [Pierre Auger], Phys. Rev. Lett. 125, no.12, 121106 (2020).

[11] R. U. Abbasi et al. [HiRes], Phys. Rev. Lett. 100, 101101 (2008).

[12] D. Bergman et al. [Telescope Array Collaboration] PoS ICRC 2021, 339 (2021).

[13] Y. Tsunesada et al. [Pierre Auger Collaboration and Telescope Array Collaboration] PoS ICRC 2021, 337 (2021).

[14] R. U. Abbasi, M. Abe, T. Abu-Zayyad, M. Allen, Y. Arai, R. Arimura, R. Azuma, E. Barcikowski, J. W. Belz and D. R. Bergman, et al. [arXiv:1801.07820 [astro-ph.HE]].

[15] T. AbuZayyad et al. [Telescope Array Collaboration] PoS ICRC 2021, 346 (2021).

[16] K. Fujita et al. [Telescope Array Collaboration] PoS ICRC 2021, 353 (2021).

[17] R. U. Abbasi et al. [Telescope Array], Astrophys. J. 909, no.2, 178 (2021).

[18] R. U. Abbasi et al. [Telescope Array], Astrophys. J. 858, no.2, 76 (2018).

[19] H.S. Shin et al. [Telescope Array Collaboration] PoS ICRC 2021, 305 (2021).

[20] Y. Zhezher et al. [Telescope Array Collaboration] PoS ICRC 2021, 300 (2021).

[21] R. U. Abbasi et al. [Telescope Array], Phys. Rev. D 99, no.2, 022002 (2019). 
[22] B. Peters Il Nuovo Cim. XXII, 800-819 (1961).

[23] T. K. Gaisser, T. Stanev and S. Tilav, Front. Phys. (Beijing) 8, 748-758 (2013).

[24] C. J. Todero Peixoto et al. [Pierre Augers Collaboration] PoS ICRC 2019, 440 (2019).

[25] D. Bergman et al. [Telescope Array Collaboration] PoS ICRC 2021, 338 (2021).

[26] R. U. Abbasi et al. [Telescope Array], Phys. Rev. D 92, no.3, 032007 (2015).

[27] I. I. Karpikov, G. I. Rubtsov and Y. V. Zhezher, Phys. Rev. D 98, no.10, 103002 (2018).

[28] R. Abbasi et al. [Telescope Array Collaboration] PoS ICRC 2021, 296 (2021).

[29] O. Kalashev et al. [Telescope Array Collaboration] PoS ICRC 2021, 864 (2021).

[30] I. Kharuk et al. [Telescope Array Collaboration] PoS ICRC 2021, 384 (2021).

[31] R. U. Abbasi et al. [Telescope Array Collaboration], Astropart. Phys. 110, 8-14 (2019).

[32] K. Shinozaki et al., Astrophys. J. 571, L117 (2002).

[33] J. Abraham et al. [Pierre Auger Collaboration], Astropart. Phys. 29, 243 (2008).

[34] J. Rautenberg et al. [Pierre Auger Collaboration], PoS, ICRC 2019, 398 (2020).

[35] A. Aab et al. [Pierre Auger Collaboration], JCAP 1704 (2017) 009.

[36] A. V. Glushkov et al., Phys. Rev. D82, 041101 (2010).

[37] G. Gelmini, O. E. Kalashev and D. V. Semikoz, J. Exp. Theor. Phys. 106, 1061 (2008).

[38] D. Hooper, A. M. Taylor and S. Sarkar, Astropart. Phys. 34, 340-343 (2011).

[39] M. Kachelriess, O. E. Kalashev and M. Y. Kuznetsov, Phys. Rev. D 98, no.8, 083016 (2018).

[40] I. Tkachev et al. [Telescope Array Collaboration] PoS ICRC 2021, 392 (2021).

[41] A. Aab et al. [Pierre Auger], Science 357, no.6537, 1266-1270 (2017).

[42] V. Verzi, D. Ivanov and Y. Tsunesada, PTEP 2017, no.12, 12A103 (2017).

[43] T. Fujii et al. [Telescope Array Collaboration] PoS ICRC 2021, 291 (2021).

[44] P. Tinyakov et al. [Pierre Auger Collaboration and Telescope Array Collaboration] PoS ICRC 2021, 375 (2021).

[45] R. U. Abbasi et al. [Telescope Array], Astrophys. J. Lett. 790, L21 (2014).

[46] J. Kim et al. [Telescope Array Collaboration] PoS ICRC 2021, 328 (2021).

[47] M. Y. Kuznetsov and P. G. Tinyakov, JCAP 04, 065 (2021). 
[48] M. Kuznetsov et al. [Telescope Array Collaboration] PoS ICRC 2021, 294 (2021).

[49] A. Aab et al. [Pierre Auger], JCAP 04, 038 (2017).

[50] R. U. Abbasi et al. [Telescope Array Project], Phys. Lett. A 381, no.32, 2565-2572 (2017).

[51] R. U. Abbasi, T. Abu-Zayyad, M. Allen, R. Azuma, E. Barcikowski, J. W. Belz, D. R. Bergman, S. A. Blake, M. Byrne and R. Cady, et al. J. Geophys. Res. Atmos. 123, 6864 (2018).

[52] J. Remington et al. [Telescope Array Collaboration] PoS ICRC 2021, 345 (2021).

[53] D. Heck, J. Knapp, J. N. Capdevielle, G. Schatz and T. Thouw, FZKA-6019.

[54] R. Abbasi et al. [Telescope Array Collaboration] PoS ICRC 2021, 297 (2021). 


\section{Full Authors List: Telescope Array Collaboration}

R.U. Abbasi ${ }^{1}$, T. Abu-Zayyad ${ }^{1,2}$, M. Allen ${ }^{2}$, Y. Arai ${ }^{3}$, R. Arimura ${ }^{3}$, E. Barcikowski ${ }^{2}$, J.W. Belz ${ }^{2}$, D.R. Bergman ${ }^{2}$, S.A. Blake ${ }^{2}$, I. Buckland ${ }^{2}$, R. Cady $^{2}$, B.G. Cheon ${ }^{4}$, J. Chiba ${ }^{5}$, M. Chikawa ${ }^{6}$, T. Fujii ${ }^{7}$, K. Fujisue ${ }^{6}$, K. Fujita ${ }^{3}$, R. Fujiwara ${ }^{3}$, M. Fukushima ${ }^{6}$, R. Fukushima ${ }^{3}$, G. Furlich ${ }^{2}$, R. Gonzalez ${ }^{2}$, W. Hanlon ${ }^{2}$, M. Hayashi ${ }^{8}$, N. Hayashida ${ }^{9}$, K. Hibino ${ }^{9}$, R. Higuchi ${ }^{6}$, K. Honda ${ }^{10}$, D. Ikeda ${ }^{9}$, T. Inadomi ${ }^{11}$, N. Inoue ${ }^{12}$, T. Ishii ${ }^{10}$, H. Ito ${ }^{13}$, D. Ivanov ${ }^{2}$, H. Iwakura ${ }^{11}$, A. Iwasaki ${ }^{3}$, H.M. Jeong ${ }^{14}$, S. Jeong ${ }^{14}$, C.C.H. Jui ${ }^{2}$, K. Kadota ${ }^{15}$, F. Kakimoto ${ }^{9}$, O. Kalashev ${ }^{16}$, K. Kasahara ${ }^{17}$, S. Kasami ${ }^{18}$, H. Kawai ${ }^{19}$, S. Kawakami ${ }^{3}$, S. Kawana ${ }^{12}$, K. Kawata ${ }^{6}$, I. Kharuk ${ }^{16}$, E. Kido ${ }^{13}$, H.B. Kim ${ }^{4}$, J.H. Kim ${ }^{2}$, J.H. Kim² ${ }^{2}$ M.H. Kim ${ }^{14}$, S.W. Kim ${ }^{14}$, Y. Kimura ${ }^{3}$, S. Kishigami ${ }^{3}$, Y. Kubota ${ }^{11}$, S. Kurisu ${ }^{11}$, V. Kuzmin ${ }^{16^{\dagger}}$, M. Kuznetsov ${ }^{16,20}$, Y.J. Kwon ${ }^{21}$, K.H. Lee ${ }^{14}$, B. Lubsandorzhiev ${ }^{16}$, J.P. Lundquist ${ }^{2,22}$, K. Machida $^{10}$, H. Matsumiya ${ }^{3}$, T. Matsuyama ${ }^{3}$, J.N. Matthews ${ }^{2}$, R. Mayta ${ }^{3}$, M. Minamino ${ }^{3}$, K. Mukai ${ }^{10}$, I. Myers ${ }^{2}$, S. Nagataki ${ }^{13}$, K. Nakai ${ }^{3}$, R. Nakamura ${ }^{11}$, T. Nakamura ${ }^{23}$, T. Nakamura ${ }^{11}$, Y. Nakamura ${ }^{11}$, A. Nakazawa ${ }^{11}$, E. Nishio ${ }^{18}$,

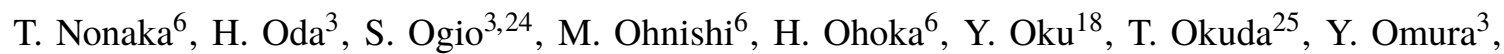
M. Ono ${ }^{13}$, R. Onogi ${ }^{3}$, A. Oshima ${ }^{3}$, S. Ozawa ${ }^{26}$, I.H. Park ${ }^{14}$, M. Potts ${ }^{2}$, M.S. Pshirkov ${ }^{16,27}$, J. Remington ${ }^{2}$, D.C. Rodriguez ${ }^{2}$, G.I. Rubtsov ${ }^{16}$, D. Ryu ${ }^{28}$, H. Sagawa ${ }^{6}$, R. Sahara ${ }^{3}$, Y. Saito ${ }^{11}$, N. Sakaki ${ }^{6}$, T. Sako ${ }^{6}$, N. Sakurai ${ }^{3}$, K. Sano ${ }^{11}$, K. Sato ${ }^{3}$, T. Seki ${ }^{11}$, K. Sekino ${ }^{6}$, P.D. Shah ${ }^{2}$, Y. Shibasaki ${ }^{11}$, F. Shibata ${ }^{10}$, N. Shibata ${ }^{18}$, T. Shibata ${ }^{6}$, H. Shimodaira ${ }^{6}$, B.K. Shin ${ }^{28}$, H.S. Shin ${ }^{6}$, D. Shinto ${ }^{18}$, J.D. Smith ${ }^{2}$, P. Sokolsky ${ }^{2}$, N. Sone ${ }^{11}$, B.T. Stokes ${ }^{2}$, T.A. Stroman ${ }^{2}$, Y. Takagi ${ }^{3}$,

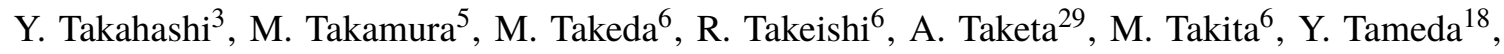

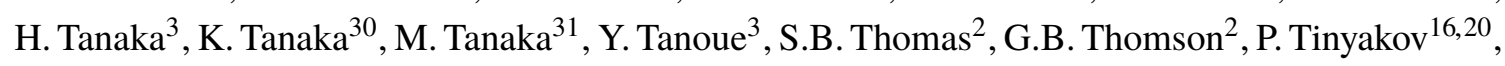
I. Tkachev ${ }^{16}$, H. Tokuno ${ }^{32}$, T. Tomida ${ }^{11}$, S. Troitsky ${ }^{16}$, R. Tsuda ${ }^{3}$, Y. Tsunesada ${ }^{3,24}$, Y. Uchihori ${ }^{33}$, S. Udo ${ }^{9}$, T. Uehama ${ }^{11}$, F. Urban ${ }^{34}$, T. Wong ${ }^{2}$, K. Yada ${ }^{6}$, M. Yamamoto ${ }^{11}$, K. Yamazaki ${ }^{9}$, J. Yang ${ }^{35}$, K. Yashiro ${ }^{5}$, F. Yoshida ${ }^{18}$, Y. Yoshioka ${ }^{11}$, Y. Zhezher ${ }^{6,16}$, and Z. Zundel ${ }^{2}$

${ }^{1}$ Department of Physics, Loyola University Chicago, Chicago, Illinois, USA

${ }^{2}$ High Energy Astrophysics Institute and Department of Physics and Astronomy, University of Utah, Salt Lake City, Utah, USA

${ }^{3}$ Graduate School of Science, Osaka City University, Osaka, Osaka, Japan

${ }^{4}$ Department of Physics and The Research Institute of Natural Science, Hanyang University, Seongdong-gu, Seoul, Korea

${ }^{5}$ Department of Physics, Tokyo University of Science, Noda, Chiba, Japan

${ }^{6}$ Institute for Cosmic Ray Research, University of Tokyo, Kashiwa, Chiba, Japan

7 The Hakubi Center for Advanced Research and Graduate School of Science, Kyoto University, KitashirakawaOiwakecho, Sakyo-ku, Kyoto, Japan

${ }^{8}$ Information Engineering Graduate School of Science and Technology, Shinshu University, Nagano, Nagano, Japan

${ }^{9}$ Faculty of Engineering, Kanagawa University, Yokohama, Kanagawa, Japan

${ }^{10}$ Interdisciplinary Graduate School of Medicine and Engineering, University of Yamanashi, Kofu, Yamanashi, Japan

${ }^{11}$ Academic Assembly School of Science and Technology Institute of Engineering, Shinshu University, Nagano, Nagano, Japan

12 The Graduate School of Science and Engineering, Saitama University, Saitama, Saitama, Japan

${ }^{13}$ Astrophysical Big Bang Laboratory, RIKEN, Wako, Saitama, Japan

${ }^{14}$ Department of Physics, SungKyunKwan University, Jang-an-gu, Suwon, Korea

15 Department of Physics, Tokyo City University, Setagaya-ku, Tokyo, Japan

${ }^{16}$ Institute for Nuclear Research of the Russian Academy of Sciences, Moscow, Russia

${ }^{17}$ Faculty of Systems Engineering and Science, Shibaura Institute of Technology, Minato-ku, Tokyo, Japan 
${ }^{18}$ Department of Engineering Science, Faculty of Engineering, Osaka Electro-Communication University, Neyagawashi, Osaka, Japan

${ }^{19}$ Department of Physics, Chiba University, Chiba, Chiba, Japan

${ }^{20}$ Service de Physique Théorique, Université Libre de Bruxelles, Brussels, Belgium

${ }^{21}$ Department of Physics, Yonsei University, Seodaemun-gu, Seoul, Korea

22 Center for Astrophysics and Cosmology, University of Nova Gorica, Nova Gorica, Slovenia

${ }^{23}$ Faculty of Science, Kochi University, Kochi, Kochi, Japan

${ }^{24}$ Nambu Yoichiro Institute of Theoretical and Experimental Physics, Osaka City University, Osaka, Osaka, Japan

${ }^{25}$ Department of Physical Sciences, Ritsumeikan University, Kusatsu, Shiga, Japan

${ }^{26}$ Quantum ICT Advanced Development Center, National Institute for Information and Communications Technology, Koganei, Tokyo, Japan

27 Sternberg Astronomical Institute, Moscow M.V. Lomonosov State University, Moscow, Russia

${ }^{28}$ Department of Physics, School of Natural Sciences, Ulsan National Institute of Science and Technology, UNIST-gil, Ulsan, Korea

${ }^{29}$ Earthquake Research Institute, University of Tokyo, Bunkyo-ku, Tokyo, Japan

${ }^{30}$ Graduate School of Information Sciences, Hiroshima City University, Hiroshima, Hiroshima, Japan

${ }^{31}$ Institute of Particle and Nuclear Studies, KEK, Tsukuba, Ibaraki, Japan

${ }^{32}$ Graduate School of Science and Engineering, Tokyo Institute of Technology, Meguro, Tokyo, Japan

33 Department of Research Planning and Promotion, Quantum Medical Science Directorate, National Institutes for Quantum and Radiological Science and Technology, Chiba, Chiba, Japan

${ }^{34}$ CEICO, Institute of Physics, Czech Academy of Sciences, Prague, Czech Republic

${ }^{35}$ Department of Physics and Institute for the Early Universe, Ewha Womans University, Seodaaemun-gu, Seoul, Korea

\section{Acknowledgements:}

The Telescope Array experiment is supported by the Japan Society for the Promotion of Science(JSPS) through Grantsin-Aid for Priority Area 431, for Specially Promoted Research JP21000002, for Scientific Research (S) JP19104006, for Specially Promoted Research JP15H05693, for Scientific Research (S) JP15H05741 and JP19H05607, for Science Research (A) JP18H03705, for Young Scientists (A) JPH26707011, and for Fostering Joint International Research (B) JP19KK0074, by the joint research program of the Institute for Cosmic Ray Research (ICRR), The University of Tokyo; by the Pioneering Program of RIKEN for the Evolution of Matter in the Universe (r-EMU); by the U.S. National Science Foundation awards PHY-1404495, PHY-1404502, PHY-1607727, PHY-1712517, PHY-1806797 and PHY-2012934; by the National Research Foundation of Korea (2017K1A4A3015188, 2020R1A2C1008230, \& 2020R1A2C2102800) ; by the Russian Science Foundation grant No. 17-72-20291 (INR), IISN project No. 4.4501.18, and Belgian Science Policy under IUAP VII/37 (ULB). This work was partially supported by the grants of the joint research program of the Institute for Space-Earth Environmental Research, Nagoya University and Inter-University Research Program of the Institute for Cosmic Ray Research of University of Tokyo. The foundations of Dr. Ezekiel R. and Edna Wattis Dumke, Willard L. Eccles, and George S. and Dolores Doré Eccles all helped with generous donations. The State of Utah supported the project through its Economic Development Board, and the University of Utah through the Office of the Vice President for Research. The experimental site became available through the cooperation of the Utah School and Institutional Trust Lands Administration (SITLA), U.S. Bureau of Land Management (BLM), and the U.S. Air Force. We appreciate the assistance of the State of Utah and Fillmore offices of the BLM in crafting the Plan of Development for the site. Patrick A. Shea assisted the collaboration with valuable advice and supported the collaboration's efforts. The people and the officials of Millard County, Utah have been a source of steadfast and warm support for our work which we greatly appreciate. We are indebted to the Millard County Road Department for their efforts to maintain and clear the roads which get us to our sites. We gratefully acknowledge the contribution from the technical staffs of our home institutions. An allocation of computer time from the Center for High Performance Computing at the University of Utah is gratefully acknowledged. The cluster of the Theoretical Division of INR RAS was used for the numerical part of the work. The lightning data used in this paper was obtained from Vaisala, Inc. We appreciate Vaisala's academic research policy.

\footnotetext{
${ }^{\dagger}$ Deceased
} 